\begin{tabular}{|c|c|c|}
\hline ITC 2/47 & \multicolumn{2}{|c|}{$\begin{array}{c}\text { A New Fuzzy Version of the Risk-constrained Cash-in-Transit } \\
\text { Vehicle Routing Problem }\end{array}$} \\
\hline $\begin{array}{l}\text { Vol. 47/ No. 2/2018 } \\
\text { pp. 321-337 }\end{array}$ & Received 2018/01/09 & Accepted after revision 2018/05/07 \\
\hline 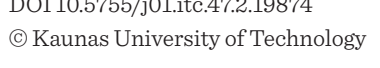 & \multicolumn{2}{|c|}{ eross } \\
\hline
\end{tabular}

\title{
A New Fuzzy Version of the Risk-constrained Cash-in-Transit Vehicle Routing Problem
}

\section{Nina Radojičić, Miroslav Marić}

Faculty of Mathematics, University of Belgrade; Studentski trg 16, 11000 Belgrade, Serbia; e-mails:nina@matf.bg.ac.rs; maricm@matf.bg.ac.rs

\author{
Aleksandar Takači \\ Faculty of Technology, University of Novi Sad; Bulevar cara Lazara 1, 21000 Novi Sad, Serbia; \\ e-mail: stakaci@tf.uns.ac.rs
}

\section{Corresponding author: nina@matf.bg.ac.rs}

This paper considers the Risk-constrained Cash-in-Transit Vehicle Routing Problem (RCTVRP), a variant of the vehicle routing problem which takes into account risk factors of the routes. In this problem, the risk constraints are set by using a risk threshold $\mathrm{T}$ on each route and thus, the routes with risk larger than $\mathrm{T}$ are forbidden. The main idea of this paper is to use the possibility of being robbed along each route, instead of just allowing solutions with routes that satisfy the risk constraints. We develop a new fuzzy version of the RCTVRP, called FRCTVRP, which considers the value of the risk index of each route and the solutions with lower values of risk indexes on their routes are considered as better. In order to achieve that, fuzzy numbers are incorporated into the new formulation. Moreover, two mixed integer program formulations of the FRCTVRP are developed in the paper. The introduced FRCTVRP is compared with the classical RCTVRP from the literature on an adequate example and the advantage of the newly proposed FRCTVRP is demonstrated. Computational experiments are performed and the comparison given in the paper shows that our approach leads to safer routes.

KEYWORDS: Combinatorial Optimization, Vehicle Routing Problem, Security, Cash-in-Transit, Fuzzy Modeling. 


\section{Introduction}

The vehicle routing problems (VRPs) have a considerably wide range of applications, especially in the fields of physical distribution and logistics, and as a result have been attracting many researchers. VRPs are considered as one of the major classes of combinatorial optimization problems and can be described as the problems of designing optimal delivery or collection routes from one or several depots to a number of customers, subject to some additional constraints depending on the application. There exists a wide variety of VRPs and a broad literature on this class of problems, such as the vehicle routing problem with time windows [1, 6] and multi-depot vehicle routing problems [5]. Classical VRP can be seen as a generalization of the travelling salesman problem (TSP) which is one of the most widely studied combinatorial optimization problems $[2,16]$. A literature review regarding VRPs can be found in [11], while more insights on the latest advances and challenges on VRPs are available in [9].

The Risk-constrained Cash-in-Transit Vehicle Routing Problem (RCTVRP), first introduced in [19], represents a special case of the vehicle routing problem. In addition to the classical aim to minimize the travel time or cost, this problem also takes into account the safety aspects of the routes. Reducing the threat of a robbery is an important issue for transportation companies that work in the cash-in-transit sector. One of the important areas to work on is creating routes thoughtfully in order to increase safety. In the literature, there are some ideas about creating routes that are not easy to predict, specifically the so-called m-peripatetic vehicle routing problem [17]. In this problem, customers are visited more than once, but the same road between two customers can not be used twice.

However, in addition to meeting safety requirements, routing plans in the cash-in-transit sector should be efficient in the sense of travel time/cost. The RCTVRP, developed in [19], considers limiting the exposure of the valuable goods to risk during transportation, while minimizing the overall time/cost of travel along all the routes. In the RCTVRP, they introduce the criteria that the total risk on each route cannot exceed a constant called the risk threshold. The same problem is considered in [20], where Talarico et al. proposed a large neighbourhood metaheuristic for solving the RCTVRP.

\subsection{Motivation for Fuzzy Models}

It is well known that real problems often contain some degree of uncertainty. It is not rare that the explanation of some real problems contain linguistic vagueness perfectly understandable to human beings. Despite the comprehensiveness of the linguistic explanation of the problem, these problems remain challenging to be precisely formulated by using classical mathematical approaches.

The following two examples are considered comprehensive within natural language: "distance is about 5 kilometers" and "daily profit is between 10 and 20 thousand". While creating a model where these values have to be represented as real numbers, one would probably write values $5 \mathrm{~km}$ and 15000 . However, the optimal solution obtained on the model generated with these strict values might be significantly different from the optimal solution of the original problem where the proper values are $5.02 \mathrm{~km}$ and 12359 .

Zadeh, the originator of fuzzy logic, proposed the idea of applying natural language terms in the realm of fuzzy logic [23]. Since then, many real problems have been formulated by using fuzzy concepts. Fuzzy logic has been successfully applied in various fields, from addressing uncertainties when reducing the size of a text [12] to modeling various optimization problems [7].

When creating a mathematical formulation of a problem, different constraints are often introduced. For example, in VRPs, each customer should be visited exactly once. However, not everything can be described using binary sets. The classifications of customers into visited and not visited is easy, but it is challenging to classify the route as being safe or not safe. The set of safe routes is far more difficult to define, because there is no distinct cut-off point at which routes stop being safe. Even measuring the risks of the routes more precisely would not solve this issue completely. However, if a risk degree is added to each route, then the routes can be ranked based on their safety degree. Fuzzy sets are ideal to model this degree.

Commonly in the literature, fuzzy sets theory has been used in order to deal with imprecise observed data or parameters. There is a whole area in operation research, called fuzzy linear programming (FLP) and 
some of the most common models and procedures for solving FLP problems were presented and analyzed in [8]. In addition, integer linear programming problems in fuzzy environment have been studied. Three models based on fuzzy number ranking methods were presented in [13]. However, in this paper, we construct a variation of the RCTVRP by using a fuzzy set theory and then provide its mixed integer programming formulation enabling it to be solved by the same techniques as performed on the non-fuzzy version of the problem.

\subsection{A Review of the Literature Related to Fuzzy VRP Models}

In order to model various variants of VRPs, fuzzy logic has been used in the literature as an interesting approach to deal with the challenge of constructing more realistic mathematical models for real world problems. Many papers consider the VRPs with fuzzy demands where the situation when the demands at nodes are uncertain. Teodorović and Pavković [22] changed classical VRP by using a triangular fuzzy number to represent a demand at a node, since they assumed that the quantities to be collected at the node are only approximately known. In a similar way, fuzzy demands are combined with a version of VRP, where a vehicle is not required to return to the distribution depot after servicing the last customer on its route, the open vehicle routing problem (OVRP). The OVRP is considered in [4], where a fuzzy chance-constrained program model is designed based on fuzzy credibility theory. Additionally, in [15], fuzzy demands for a variant of VRP are considered and a case study on a garbage collection system is performed.

Moreover, VRPs with fuzzy time windows are considered in [21]. Since time windows are not always strictly obeyed, Tang et al. [21] applied fuzzy membership functions to characterize the service level issues associated with time window violation in a vehicle routing problem and proposed a problem formulated as a multi-objective model with two goals: to minimize the travel distance and to maximize the service level of the supplier to customers. The other paper [10] considers a multi-objective dynamic vehicle routing problem with fuzzy time windows (DVRPFTW), where a set of real time requests arrives randomly over time. Since vehicles are routed according to customer-specific time windows, which are highly relevant to the customers' satisfaction level, Ghan- nadpour et al. [10] represented this preference information of customers as a fuzzy number with respect to the satisfaction for a service time. Moreover, Brito et al. [3] studied the close-open vehicle routing problem, which is a variant of VRP, where there is no requirement that all the vehicles have to return to the depot after completing their service. In that paper, the capacity and time windows constraints are considered flexible and modeled as fuzzy constraints, since customer demands and travel times in real world situations are imprecise.

Until now, there is no published work yet that applies fuzzy theory to model the RCTVRP.

\subsection{Contributions}

The main contribution of this paper is a new fuzzy variant of the RCTVRP for more adequate modeling of safe routes. Note that, in the model from [19], the risk constraints are satisfied if for each route the global risk index is not larger than a predefined threshold $\mathrm{T}$, which is an input data. However, these constraints do not take into account how much smaller than $\mathrm{T}$ the risk indexes are. The focus of this paper is how to use the imprecision (fuzziness) in order to create a model that is more accurate in terms of the risk threshold constraints. The idea is that each risk constraint can be satisfied to a certain degree, i.e. we differentiate if the global risk is "much smaller" than $\mathrm{T}$ or if it is near the threshold $\mathrm{T}$ in order to be able to prefer the safer routes. In the paper, this approach was shown to be beneficial in producing routes with a lower possibility of being robbed.

In order to represent risk constraints in a more realistic way, we propose fuzzy numbers based on the threshold constant $T$ and the risk indexes of the routes and incorporate them into the objective function. Note that previously listed literature on fuzzy VRPs have dealt with fuzzification of some of the uncertain input data (e.g. distances, demands and time windows), while we use a fuzzy number to model the risk factor conditions in a better way.

Moreover, two mixed integer program formulations of the newly proposed fuzzy RCTVRP are developed in the paper. To present the advantage of our approach, the new fuzzy model is compared to the model from the literature [19] on an example. Furthermore, a comparison is performed on appropriate data sets and the computational results are presented. The 
obtained solutions showed that our fuzzy version of the problem leads to better solutions in terms of safety and is thus better suited for real-life situations in cash-in-transit sector.

\section{Definitions and Preliminaries}

This section contains some of the main definitions from fuzzy set theory, mainly taken from [24]. More on this topic can also be found in [14]. Let $X$ be a collection of objects, with a generic element of set $X$ de$\operatorname{noted} x$.

Definition 1. [Fuzzy set] A fuzzy set $\tilde{A}$ is a set of ordered pairs $\tilde{A}=\left\{\left(x, \mu_{\tilde{A}}(x)\right) \mid x \in \mathrm{X}\right\}$. In the pair $\left(x, \mu_{\tilde{A}}(x)\right)$, the first element $x$ belongs to the classical set $X$, the second element $\mu_{\hat{A}}$ is called the membership function or grade function of $x$ in $\tilde{A}$ that maps $X$ to the membership space $M$.

Usually, the interval $[0,1]$ is used as $M$. Note that in the case when $M$ contains only the two points 0 and 1 , $\tilde{A}$ is a classical set and $\mu_{\tilde{A}}$ is identical to the characteristic function of a classical set. The range of the membership function is a subset of the nonnegative real numbers whose supremum is finite. Elements with a zero degree of membership are normally not listed.

The membership function fully defines the fuzzy set. A membership function provides a measure of the degree of similarity of an element to a fuzzy set, which allows many applications. Membership functions can take any form, but there are some common examples that appear in real applications due to their suitability for representing uncertain information.

Definition 2. The support of a fuzzy set $\tilde{A}, S(\tilde{A})$, is the crisp set of all $x \in X$ such that $\mu_{\tilde{A}}(x)>0$.

Definition 3. A fuzzy set $\tilde{A}$ is normal if there is at least one point $x \in \mathbb{R}$ with $\mu_{\tilde{A}}(x)=1$.

Definition 4. A fuzzy set $\tilde{A}$ is convex if $\mu_{\tilde{A}}\left(\lambda x_{1}+\right.$ $\left.(1-\lambda) x_{2}\right) \geq \min \left(\mu_{\tilde{A}}\left(x_{1}\right), \mu_{\hat{A}}\left(x_{2}\right)\right.$, for every $x_{1}, x_{2} \in \mathrm{X}$ and $\lambda \in[0,1]$.

Definition 5. [Fuzzy number] A fuzzy number $\tilde{M}$ is a convex normalized fuzzy set $\tilde{M}$ of real line $\mathbb{R}$ such that $\mu_{\hat{A}}(x)$ is piecewise continuous.

A fuzzy number is a generalization of a regular, real number in the sense that it does not refer to one single value but rather to a connected set of possible values, where each possible value has its own weight between 0 and 1 . This weight is called the membership function. There are various possibilities. In this paper, we use the left shoulder fuzzy number presented in Figure 1, and its membership function can be represented as

$$
\mu(x)=\left\{\begin{aligned}
0, & \text { if } x<c \\
\frac{x-c}{x-d,}, & \text { if } c \leq x<d \\
1, & \text { otherwise } .
\end{aligned}\right.
$$

\section{Figure 1}

The left shoulder fuzzy number has an increasing piecewise linear membership function that connects the points $(-\infty, 0)$, $(c, 0),(d, 1)$ and $(\infty, 1)$

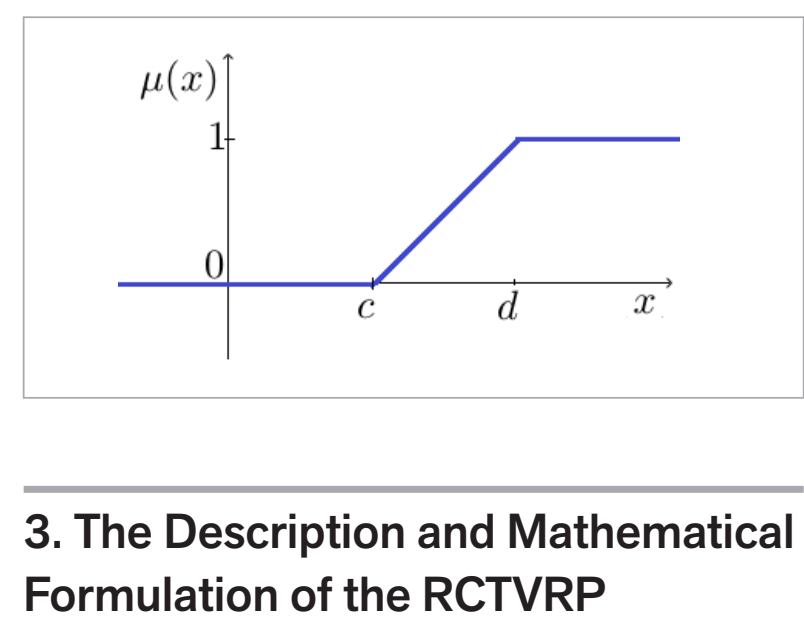

The RCTVRP is stated as follows: a depot and a set of customers (e.g. supermarkets, shopping centers, clothes shops, jewelry stores) with their demands are given. Each customer should be visited once and only once by exactly one vehicle and the demand of each customer must be completely collected by a single vehicle. Each vehicle leaves the depot empty, visits the customers along the route, collects the goods and finally drops the collected goods off at the same depot. The exposure of the goods to risks (e.g. robberies) should be limited.

\subsection{Measuring the Risk Along the Route}

Safety aspects in transportation have been studied in the literature from different perspectives. In order to create a mathematical model for the desired problem, numerical values for the risk levels along the roads would be helpful. The notation is given in the follow- 
ing manner: here and subsequently, $R_{j}^{r}$ stands for the risk level along the route $r$ for the vehicle before arriving at node $j$, while $p_{i j}$ denotes the probability that a robbery happens on the $\operatorname{arc}(i, j)$.

As presented in [19], since a robbery might happen along the arc $(i, j)$, if the vehicle moves from node $i$ to node $j$ along the route $r$, the risk on the route $r$ along the $\operatorname{arc}(i, j)$ can be calculated as $R_{j}^{r}=p_{i j} \cdot v_{i j} \cdot D_{i}^{r}$. In the previous formula, $v_{i j}$ represents the vulnerability, i.e. a measure that the robbery succeeds given it occurs and $D_{i}^{r}$ stands for the value of goods inside of the vehicle after the visit of node $i$ along the route $r$. Note that in some applications, the adequate values for $p_{i j}$ and $v_{i j}$ are not known. Thus, $p_{i j}$ can be replaced with the distance $c_{i j}$ between nodes $i$ and $j$, while $v_{i j}$ can be set to 1 . For simplicity, this is assumed in the rest of the paper. Moreover, since the probability a robbery occurs more than once along the same route is small, it can be assumed for the sake of simplicity that the robbery cannot happen twice or more along the same route. More details on measuring the risk along the route can be found in [19].

Finally, since each vehicle starts empty from the depot, collects cash along the route and delivers it only to the depot at the end of route, $D_{0}^{r}=0$ and the amount of money increases along the route. The risk index also increases along the route and can be calculated for each node $j$ where the vehicle arrives from the node $i$ along the route $r$ by using the following recursive formula

$$
R_{j}^{r}=R_{i}^{r}+D_{i}^{r} \cdot c_{i j} .
$$

Note that $R_{0}^{r}=0$ i.e. starting from depot 0 the risk index is equal to zero.

\subsection{Mathematical Formulation}

In this paper, we start from a mixed-integer formulation of the RCTVRP introduced in [19].

Let $N=\{1,2, \ldots, n\}$ be the set of customers that should be visited. The amount of cash that should be collected from the $i \in N$ is denoted by $d_{i}$. In the model, the depot is, for simplicity's sake, represented with two nodes $s$ (start) and $e$ (end), with zero demands $\left(d_{s}=\right.$ $\left.d_{e}=0\right)$.

Thus, the problem is defined on a directed graph $G=$ $(V, A)$, where $V=N \cup\{s, e\}$ and $A=(N \times N) \cup(\{s\} \times N) \cup$
$(N \times\{e\})$. Each arc $(i, j) \in$ A has its own constant $c_{i j}$ that represents distance or travel time between nodes $i$ and $j$. Each vehicle starts from s without any cash loaded and thus has a risk index equal to zero. Along the route, both the amount of cash and the risk index increase and the mixed integer formulation from [19] contains two adequate families of decision variables. In what follows, $D_{i}^{r}$ stands for the amount of cash carried by the vehicle when it leaves node $i$ along the route $r$, while $R_{i}^{r}$ represents the risk index for the vehicle when it arrives at node $i$ along the route $r$. In this RCTVRP formulation, each cumulative measure (i.e. $D_{i}^{r}$ and $R_{i}^{r}$ ) is a part of a constraint and is not taken into account in the objective function.

The binary decision variable $x_{i j}^{r}$ is defined as:

$$
x_{i j}^{r}=\left\{\begin{array}{ll}
1, & \text { if arc }(i, j) \in A \text { is traversed } \\
& \text { by the vehicle along route } r
\end{array},\right.
$$

In the RCTVRP formulation from [19], the objective function is the total travel length/costs along all the routes. The goal is to determine the routes (as well as the number of routes) so that the objective function is minimized. Note that the number of routes cannot be larger than $n$ (the worst case is when each customer is visited by a different vehicle). Thus, the set $N$ is used for the indexes of routes.

Using the notation mentioned above, the problem can be written as:

$$
\min \sum_{r \in N} \sum_{(i, j) \in A} c_{i j} x_{i j}^{r}
$$

subject to:

$$
\sum_{j \in N} x_{s j}^{r}=\sum_{i \in N} x_{i e}^{r}, \forall r \in N
$$

$$
\sum_{j \in N} x_{s j}^{1}=1,
$$

$$
\sum_{i \in N} x_{i e}^{r} \geq \sum_{j \in N} x_{s j}^{r+1}, \forall r \in N \backslash\{n\},
$$

$$
\sum_{r \in N} \sum_{j \in V \backslash\{s\}} x_{i j}^{r}=1, \forall i \in N,
$$




\begin{tabular}{l}
\hline$\sum_{h \in V \backslash\{e\}} x_{h j}^{r}-\sum_{k \in V \backslash\{s\}} x_{j k}^{r}=0, \forall j \in N ; \forall r \in N$ \\
\hline$D_{s}^{r}=0, \forall r \in N$, \\
$D_{j}^{r} \geq D_{i}^{r}+d_{j}-\left(1-x_{i j}^{r}\right) \cdot M_{1}$, \\
$\forall(i, j) \in A ; \forall r \in N$, \\
\hline $0 \leq D_{i}^{r} \leq M_{1}, \forall i \in V ; \forall r \in N$, \\
$R_{s}^{r}=0, \forall r \in N$, \\
\hline$R_{j}^{r} \geq R_{i}^{r}+D_{i}^{r} \cdot c_{i j}-\left(1-x_{i j}^{r}\right) \cdot M_{2}$, \\
$\forall(i, j) \in A ; \forall r \in N$, \\
\hline $0 \leq R_{i}^{r} \leq T, \forall i \in V ; \forall r \in N$, \\
$x_{i j}^{r} \in\{0,1\}, \forall(i, j) \in A ; \forall r \in N$, \\
\hline
\end{tabular}

where $x_{i j}^{r}, D_{i}^{r}$ and $R_{i}^{r}$ are variables of the optimization problem.

The summarized length of all arcs traversed along any of the routes should be minimized (see the objective function (1). Due to the conditions (2) each route starts and ends at the depot. Condition (3) imposes that the first route (indexed with $r=1$ ) exists and starts from the depot. The route $r+1$ does not exist if $r$ does not exist, due to the constraints (4). Thus, the routes are numerated consecutively. Each customer must be visited exactly once, according to the constraints (5). In each route $r$, the vehicle exits the customer $j$ if and only if it has enter the customer $j$ along the route $r$, due to the constraints (6). The constraints (7)-(9) set the adequate values for the variables $D_{i}^{r}$, where $M_{1}$ is a sufficiently large number $\left(M_{1}=\sum_{i \in V} d_{i}\right)$. The constraints (10)-(12) define the risk index values $R_{i}^{r}$ and impose that the global risk of each route $r$ cannot be larger than $T$, where $M_{2}$ is a sufficiently large num$\operatorname{ber}\left(M_{2}=M_{1} \cdot T \cdot\right.$ maxDistance,where maxDistance = $\max _{i, j \in V} c_{i j}$ ). Note that constant $M_{2}=T$ (used in [19]) is not enough, since it leads to disallowance of some of the feasible solutions. In fact, in the case of $x_{i j}^{r}=0$, the right hand side of equation (11) can be nonnegative and lead to falsely not satisfying one of the threshold conditions (12).
Note that subroutes are avoided, since $D_{i}^{r}$ and $R_{i}^{r}$ are increasing along each route $r$. Moreover, if the real data regarding the probability of a robbery on the arc $(i, j)$ is available, in the conditions (11) $c_{i j}$ can be replaced with $p_{i j}$.

\section{A Fuzzy Version of the RCTVRP}

In this section, we introduce a new fuzzy version of the RCTVRP, named FRCTVRP, which allows us to more precisely distinguish high-quality solutions. We follow the same notation as above and aim to keep the model as similar as possible to the presented model from [19]. A new form of the objective function is introduced and the adequate fuzzy number is included in order to create a new promising fuzzy model.

In the previously described RCTVRP, the calculated risk of each route lower than the risk threshold is not taken into further consideration. That approach simply follows the problem description where the risk cannot exceed the value. However, what is implied within the description of the risk indexes is that the lower the risk index is the safer the route is. The set of safe routes can be appropriately represented by using fuzzy sets instead of classic crisp sets. Furthermore, one approach to incorporating this fact into the model is to include it into the objective function. Since the RCTVRP is a minimization problem, instead of just minimizing the overall travel distance, a new measure for the solution quality should include both distances and the level how safe the routes are. In order to ensure that each calculated risk index has an adequate influence in a newly proposed version of the problem, the following objective function is introduced:

$$
\min \sum_{r \in N} \sum_{(i, j) \in A} c_{i j} x_{i j}^{r} F\left(R_{j}^{r}\right) .
$$

Function $F$ that is added as a part of the objective function is defined as:

$F(r)=1+\mu(r)$,

where function is the membership function of the left shoulder fuzzy number represented in Figure 2.

For the purpose of this paper, the parameter $t(t<T)$ 
Figure 2

Left shoulder fuzzy number

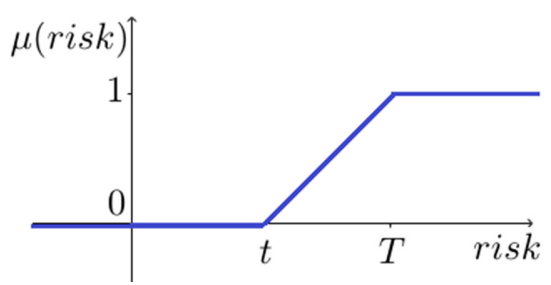

is chosen to be $t=\max _{\mathrm{i} \in \mathrm{N}} \mathrm{c}_{\mathrm{ie}} \mathrm{d}_{\mathrm{i}}$, since it seems fair to consider all routes with a single customer to be routes with minimal risk, i.e. the routes with the lowest value of the function $F$. Routes with the risk index that exceeds the risk threshold value $T$ are still forbidden. Function $\mu$ maps the risk indexes of routes to numbers from the interval $[0,1]$.

This type of membership function is chosen to represent the property "the smaller is better" that we aimed to incorporate into the objective function. In essence, the objective function is changed so that the lengths of arcs within "risky" routes are increased by multiplying by a factor between 1 and 2 . Thus, by minimizing the objective function among the routes with the same lengths safer routes are prioritized.

Note that other increasing functions could be tested as well and thus the presented model can be changed depending on the application. For example, it is possible to make a version where we differentiate the risk indexes only among the routes with the same travel distances. However, in this paper, we use the presented objective function since the aim is the compromise between overall travel distance and risk indexes, in the sense that it is acceptable to make a route a bit longer if it is a lot safer. Moreover, the choice of this increasing function is proved to be convenient for formulating the MIP models provided in this paper.

In order to formulate the FRCTVRP as a mixed integer program (MIP), new variables $F R_{j}^{r}$ are introduced into the model. Note that each variable $F R_{j}^{r}$ corresponds to the previously introduced value $F\left(R_{j}^{r}\right)$. The FRCTVRP formulation is as follows:

$\min \sum_{r \in N} \sum_{(i, j) \in A} c_{i j} x_{i j}^{r} F R_{j}^{r}$ subject to:

$$
\begin{aligned}
& \sum_{j \in N} x_{s j}^{r}=\sum_{i \in N} x_{i e}^{r}, \forall r \in N, \\
& \sum_{j \in N} x_{s j}^{1}=1, \\
& \sum_{i \in N} x_{i e}^{r} \geq \sum_{j \in N} x_{s j}^{r+1}, \forall r \in N \backslash\{n\},
\end{aligned}
$$

$\sum_{r \in N} \sum_{j \in V \backslash\{s\}} x_{i j}^{r}=1, \forall i \in N$,

$\sum_{h \in V \backslash\{e\}} x_{h j}^{r}-\sum_{k \in V \backslash\{s\}} x_{j k}^{r}=0, \forall j \in N ; \forall r \in N$,

$D_{s}^{r}=0, \quad \forall r \in N$,

$$
\begin{gathered}
D_{j}^{r} \geq D_{i}^{r}+d_{j}-\left(1-x_{i j}^{r}\right) \cdot M_{1}, \\
\forall(i, j) \in A ; \forall r \in N,
\end{gathered}
$$

$0 \leq D_{i}^{r} \leq M_{1}, \forall i \in V ; \forall r \in N$

$R_{s}^{r}=0, \forall r \in N$,

$$
R_{j}^{r} \geq R_{i}^{r}+D_{i}^{r} \cdot c_{i j}-\left(1-x_{i j}^{r}\right) \cdot M_{2},
$$$$
\forall(i, j) \in A ; \forall r \in N \text {, }
$$

$0 \leq R_{i}^{r} \leq T, \forall i \in V ; \forall r \in N$,

$F R_{i}^{r} \geq 1+\frac{R_{i}^{r}-t}{T-t}, i \in V \backslash\{s\} ; \forall r \in N$,

$1 \leq F R_{i}^{r} \leq 2, \forall i \in V ; \forall r \in N$,

$x_{i j}^{r} \in\{0,1\}, \forall(i, j) \in A ; \forall r \in N$,

where $x_{i j}^{r}, D_{i}^{r}, R_{i}^{r}$ and $F R_{i}^{r}$ are variables of the optimization problem.

The constraints (27) and (28) define the values of $F R_{j}^{r}$ to represent the values of $F\left(R_{j}^{r}\right)$, where $F$ is previ- 
ously described function $F(r)=1+\mu(\mathrm{r})$.

However, in this formulation, the number of variables and constraints increased, which is among main factors that increase the computational complexity of the problem. Thus, we present an improved version of the FRCTVRP model, where variables $R_{j}^{r}$ are omitted and the constraints (24)-(27) are replaced with the constraints (39)-(40) obtained by using the formula $F R_{i}^{r}=1+\frac{R_{i}^{r}-t}{T-t}$ (i.e. $\left.R_{j}^{r}=t+\left(F R_{i}^{r}-1\right) \cdot(T-t)\right)$. Note that this equality holds only when $R_{i}^{r}$ is from the interval $[t, T]$, which is true for the valid routes.

Finally, the improved FRCTVRP formulation is as follows:

$$
\min \sum_{r \in N} \sum_{(i, j) \in A} c_{i j} x_{i j}^{r} F R_{j}^{r}
$$

subject to:

$$
\begin{aligned}
& \sum_{j \in N} x_{s j}^{r}=\sum_{i \in N} x_{i e}^{r}, \forall r \in N, \\
& \sum_{i \in N} x_{s j}^{1}=1, \\
& \sum_{i \in N} x_{i e}^{r} \geq \sum_{j \in N} x_{s j}^{r+1}, \forall r \in N \backslash\{n\}, \\
& \sum_{r \in N} \sum_{j \in V \backslash\{s\}} x_{i j}^{r}=1, \forall i \in N, \\
& \sum_{h \in V \backslash\{e\}} x_{h j}^{r}-\sum_{k \in V \backslash\{s\}} x_{j k}^{r}=0, \\
& \forall j \in N ; \forall r \in N, \\
& \hline D_{s}^{r}=0, \forall r \in N, \\
& \hline D_{j}^{r} \geq D_{i}^{r}+d_{j}-\left(1-x_{i j}^{r}\right) \cdot M_{1}, \\
& \forall(i, j) \in A ; \forall r \in N, \\
& \hline 0 \leq D_{i}^{r} \leq M_{1}, \forall i \in V ; \forall r \in N, \\
& F R_{s}^{r}=\frac{T-2 t}{T-t}, \forall r \in N,
\end{aligned}
$$

$F R_{j}^{r} \geq F R_{i}^{r}+\frac{D_{i}^{r} \cdot c_{i j}-\left(1-x_{i j}^{r}\right) \cdot M_{2}}{T-t}$,

$\forall(i, j) \in A ; \forall r \in N$,

$1 \leq F R_{i}^{r} \leq 2, \forall i \in V ; \forall r \in N$,

$x_{i j}^{r} \in\{0,1\}, \forall(i, j) \in A ; \forall r \in N$,

with variables: $x_{i j}^{r}, D_{i}^{r}, R_{i}^{r}$ and $F R_{i}^{r}$.

Note that the previous two formulations (15)-(29) and (30)-(42) have equivalent solutions. In the rest of the paper, when comparing the solutions of the classical RCTVRP from [19] with the proposed fuzzy version, the formulation (15)-(29) is used and denoted with the FRCTVRP.

Another option for lowering the number of constraints in the first formulation for the FRCTVRP (15)-(29) is explained here. It is easy to see that constraints (26), (27) and (28) are equivalent with the following constraints:

$1+\frac{R_{i}^{r}-t}{T-t} \leq F R_{i}^{r} \leq 2, \forall i \in V ; \forall r \in N$,

$R_{i}^{r} \geq t, i \in V ; \forall r \in N$.

Since the number of these constraints is lower than the number of constraints (26), (27) and (28), it is better to replace constraints (26), (27) and (28) with constraints (43) and (44). Moreover, instead of constraints (43) and (44) or equivalently (26)-(27) and (28) the following constraints can be used:

This transformation is inspired by a similar suggestion given in [18].

\section{A Demonstrative Example}

The difference between the model from the literature RCTVRP and the proposed model FRCTVRP can be illustrated by the following simple setting. Let us consider the following situation. We are given a graph with four nodes (depot and three customers) as depicted in Figure 3 and the risk threshold $\mathrm{T}=160$. In the following, this example is considered in both models, RCTVRP and FRCTVRP. 


\section{Figure 3}

An example with an undirected graph with weighted edges representing the distance between nodes. Three customers denoted by numbers 1, 2 and 3 depicted as blue nodes, with their demands written in green. Depot is the node 0

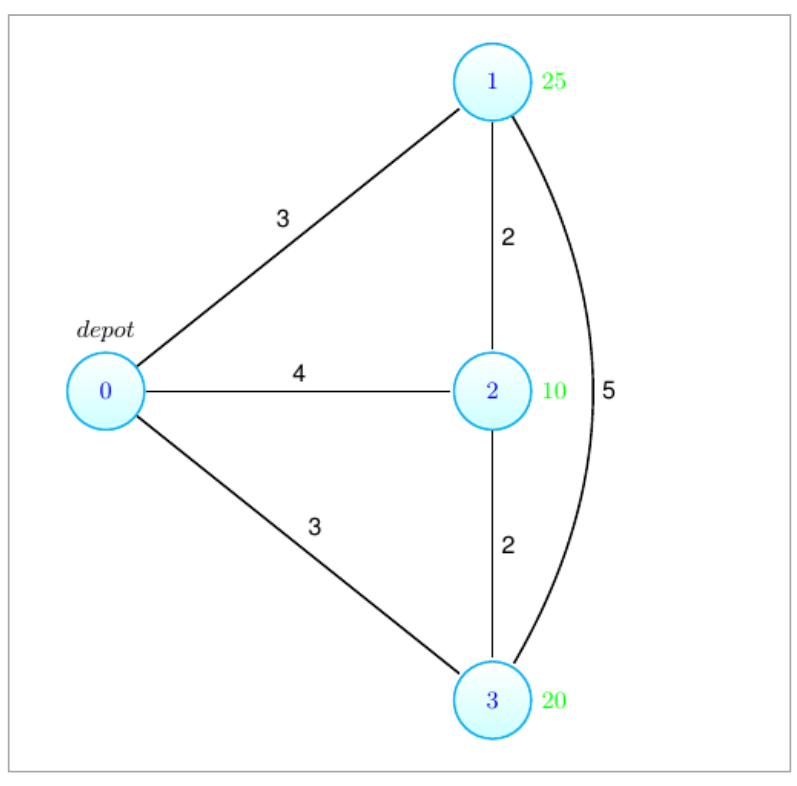

Firstly, the RCTVRP model is considered. It is easy to calculate that it is not possible to visit all three customers within one route, due to risk constraint. The solution with three routes (each customer is visited by its own vehicle) is feasible, but the objective function reaches its maximal possible value. Thus, we present more detailed calculations for the other three feasible solutions (all three with two routes each).

\section{Solution 1:}

Route $(r=1): 0 \rightarrow 2 \rightarrow 1 \rightarrow 0$

$D_{0}^{1}=0, D_{2}^{1}=d_{2}=10, D_{1}^{1}=D_{2}^{1}+d_{1}=35$,

$R_{0}^{1}=0, R_{2}^{1}=R_{0}^{1}+D_{0}^{1} \cdot c_{02}=0, R_{1}^{1}=R_{2}^{1}+D_{1}^{1}$.

$c_{21}=20$,

$G R^{1}=R_{1}^{1}+D_{1}^{1} \cdot c_{10}=125<T=160$.

Route $(r=2): 0 \rightarrow 3 \rightarrow 0$

$D_{0}^{2}=0, D_{3}^{2}=d_{3}=20$,

$R_{0}^{2}=0, R_{3}^{2}=R_{0}^{2}+D_{0}^{2} \cdot c_{03}=0$,

$G R^{2}=R_{3}^{2}+D_{3}^{2} \cdot c_{30}=60<T=160$.

\section{Solution 2:}

Route $(r=1):): 0 \rightarrow 1 \rightarrow 0$

$D_{0}^{1}=0, D_{1}^{1}=d_{1}=25$,
$R_{0}^{1}=0, R_{1}^{1}=R_{0}^{1}+D_{0}^{1} \cdot c_{01}=0$,

$G R^{1}=R_{1}^{1}+D_{1}^{1} \cdot c_{10}=75<T=160$.

Route $(r=2):): 0 \rightarrow 2 \rightarrow 3 \rightarrow 0$

$D_{0}^{2}=0, D_{2}^{2}=d_{2}=10, D_{3}^{2}=D_{2}^{2}+d_{3}=30$,

$R_{0}^{2}=0, R_{2}^{2}=R_{0}^{2}+D_{0}^{2} \cdot c_{02}=0, \quad R_{3}^{2}=R_{2}^{2}+D_{2}^{2}$.

$c_{23}=20$,

$G R^{2}=R_{3}^{2}+D_{3}^{1} \cdot c_{30}=110<T=160$

\section{Solution 3:}

Route $(r=1): 0 \rightarrow 1 \rightarrow 0$

$D_{0}^{1}=0, D_{1}^{1}=d_{1}=25$,

$R_{0}^{1}=0, R_{1}^{1}=R_{0}^{1}+D_{0}^{1} \cdot c_{01}=0$,

$G R^{1}=R_{1}^{1}+D_{1}^{1} \cdot c_{10}=75<T=160$.

Route $(r=2): 0 \rightarrow 3 \rightarrow 2 \rightarrow 0$

$D_{0}^{2}=0, D_{3}^{2}=d_{3}=20, D_{2}^{2}=D_{3}^{2}+d_{2}=30$,

$R_{0}^{2}=0, R_{3}^{2}=R_{0}^{2}+D_{0}^{2} \cdot c_{03}=0, R_{2}^{2}=R_{3}^{2}+D_{3}^{2}$.

$c_{32}=40$,

$G R^{2}=R_{2}^{2}+D_{2}^{1} \cdot c_{20}=160 \leq T=160$.

Let us note that all three previous solutions are feasible and have the same value of the objective function:

$$
\sum_{r \in N} \sum_{(i, j) \in A} c_{i j} x_{i j}^{r}=15
$$

Therefore, according to the model from [19], all three noted above solutions have the same quality. However, from the safety point of view, Solution 3 is the worst since the global risks on the two routes are $G R_{1}=75$ and $G R_{2}=160$. Preferably, a good model should differentiate the values of the objective function of solutions with the same total travel distance and different risk values.

Let us demonstrate the same example in combination to the newly presented model FRCTVRP. All the constraints remain the same, so we will discuss the same feasible solutions. The objective function stays the same in the case of the "star" solution, due to the definition of the function $F$ and the parameter $t$. Moreover, let us calculate the values of the objective function for the three solutions. Again, all three solutions have two routes each.

Solution 1: Two routes $0 \rightarrow 2 \rightarrow 1 \rightarrow 0$ and $0 \rightarrow 3 \rightarrow 0$ lead to the following value of the objective function: 


$$
\sum_{r \in N} \sum_{(i, j) \in A} c_{i j} x_{i j}^{r} F\left(R_{j}^{r}\right)=16.765
$$

Solution 2: For two routes $0 \rightarrow 1 \rightarrow 0$ and $0 \rightarrow 2 \rightarrow 3 \rightarrow 0$, we obtain:

$$
\sum_{r \in N} \sum_{(i, j) \in A} c_{i j} x_{i j}^{r} F\left(R_{j}^{r}\right)=16.235 .
$$

Solution 3: The calculation for the two routes $0 \rightarrow 1 \rightarrow 0$ and $0 \rightarrow 3 \rightarrow 2 \rightarrow 0$ is as follows

$$
\sum_{r \in N} \sum_{(i, j) \in A} c_{i j} x_{i j}^{r} F\left(R_{j}^{r}\right)=19 .
$$

Our example demonstrates that the presented FRCTVRP model leads to preferring solutions with less risky routes. When using the RCTVRP model, there are three solutions presented that have the same value of the objective function. Now, when using the FRCTVRP model, the same three solutions have three different values of the objective function. The second solution is the best, since it is slightly better than the first one. The solution number three has the largest value of the objective function among the three interesting solutions, which is expected due riskier routes used in the solution.

Note that the newly presented model FRCTVRP incorporates the possibility of a vehicle being robbed along each route, as well as keeping the good characteristic of the previous model to limit the risk on each route in a solution.

\section{Computational Results}

The example in the previous section showed how the proposed fuzzy model prefers solutions with less risky routes among the solutions with the same overall travel cost. Larger instances bring more possibilities and comparison between fuzzy and non-fuzzy version of the problem becomes more interesting. Thus, comparison tests are performed by using optimization package CPLEX 12.6. The classical RCTVRP model from [19] is compared with the proposed FRCTVRP model (15)-(29). Both models were implemented in C\# language, .NET framework, and run on an IntelCore ir7-860 2.8 GHz with 8GB RAM memory under the Windows 7 Professional operating system.

Computational experiments were firstly performed on smaller instances from a data set $\mathrm{R}$ specially constructed for the RCTVRP and described in [19]. The data set $R$ contains randomly generated instances for the RCTVRP. Basic instances with a different number of nodes $(4,6$, and 8$)$ are generated in such a way that coordinates are randomly selected from the interval $[-20,20]$. Each basic instance is combined with five different risk levels and four different values for the standard deviation of a demand vector $\left(\sigma_{d}\right)$. The first value for the risk level is defined as $R L 1=\max _{\{i \in N\}}$ $\left\{d_{i} \cdot\right.$ cie $\}$, while the others (RL1.5, RL2, RL2.5 and RL3) are generated, starting from RL1 by using an increasing multiplicative factor in steps of 0.5 , up to 3 . The demand associated with each node is generated in such a way that the standard deviation of the demand $\sigma_{d}$ is equal to $1,4,16$ or 64 .

CPLEX has been used to solve considered instances to optimality for both models and results are presented in Table 1. In the first column, the instance name is given, the second column presents the objective function value of the optimal solutions for the RCTVRP model, followed by the routes of the solution (ordered list of nodes to be visited in each route are separated by "|"), while the fourth column shows the execution time in seconds. Moreover, the last three columns contain the same values for the FRCTVRP model. For example, the fifth row shows that, for the instance "4_1_3.0", the optimal solution for the RCTVRP has the value of objective function equal to 286.1275 and there are two routes in that solution: the first route contains nodes 3 and 1 , while the second route contains only one node: 2 . The same row shows that CPLEX achieved that results within 0.2 seconds, while for the solution for the FRCTVRP it was performed in 0.09 seconds. Moreover, it can be seen in the same row that for the FRCTVRP the optimal solution has the objective function value equal to 335.2504 , and although the order of routes is not the same, these two solutions are, in fact, the same. It is important to note that the objective function is not the same and thus the two models cannot be compared by using these objective values. Therefore, we compare the results according to the nodes in routes, i.e. if the lists of nodes are the same, the results obtained for the RCRVRP and the FRCRVRP are considered the same. Note that these results have the same overall travel distance. 
Table 1

A comparison of the RCTVRP and FRCTVRP on instances from the set R

\begin{tabular}{|c|c|c|c|c|c|c|}
\hline \multirow{2}{*}{$\begin{array}{c}\text { Instance } \\
\text { name }\end{array}$} & \multicolumn{3}{|c|}{ RCTVRP } & \multicolumn{3}{|c|}{ FRCTVRP } \\
\hline & Obj Value & Nodes in routes & Time[s] & Obj Value & Nodes in routes & Time[s] \\
\hline 4_1_1.0 & 343.0474 & $3|1| 2 \mid$ & 0.06 & 343.0474 & $\mathbf{3}|\mathbf{2}| \mathbf{1} \mid$ & 0.04 \\
\hline 4_1_1.5 & 343.0474 & $3|1| 2 \mid$ & 0.06 & 343.0474 & $\mathbf{3}|\mathbf{2}| \mathbf{1} \mid$ & 0.06 \\
\hline 4_1_2.0 & 343.0474 & $3|1| 2 \mid$ & 0.29 & 343.0474 & $\mathbf{2}|\mathbf{3}| \mathbf{1} \mid$ & 0.18 \\
\hline 4_1_2.5 & 343.0474 & $3|1| 2 \mid$ & 0.07 & 343.0474 & $\mathbf{2}|\mathbf{3}| \mathbf{1} \mid$ & 0.06 \\
\hline 4_1_3.0 & $286.12^{r 75}$ & $3->1|2|$ & 0.20 & 331.07ryry & $2|3->1|$ & 0.09 \\
\hline 4_3_1.0 & 343.0474 & $3|1| 2 \mid$ & 0.05 & 343.0474 & $\mathbf{3}|\mathbf{2}| \mathbf{1} \mid$ & 0.05 \\
\hline 4_3_1.5 & 343.0474 & $3|1| 2 \mid$ & 0.05 & 343.0474 & $\mathbf{3}|\mathbf{2}| \mathbf{1} \mid$ & 0.04 \\
\hline 4_3_2.0 & 343.0474 & $3|1| 2 \mid$ & 0.23 & 343.0474 & $\mathbf{2}|\mathbf{1}| \mathbf{3} \mid$ & 0.17 \\
\hline 4_3_2.5 & 343.0474 & $3|1| 2 \mid$ & 0.07 & 343.0474 & $2|\mathbf{1}| 3 \mid$ & 0.06 \\
\hline 4_3_3.0 & 286.1275 & $3->1|2|$ & 0.30 & 333.2687 & $3->1|2|$ & 0.05 \\
\hline 4_5_1.0 & 343.0474 & $3|1| 2 \mid$ & 0.05 & 343.0474 & $\mathbf{2}|\mathbf{1}| \mathbf{3} \mid$ & 0.04 \\
\hline 4_5_1.5 & 343.0474 & $3|1| 2 \mid$ & 0.19 & 343.0474 & $1|3| 2 \mid$ & 0.16 \\
\hline 4_5_2.0 & 343.0474 & $3|1| 2 \mid$ & 0.07 & 343.0474 & $\mathbf{1}|\mathbf{3}| \mathbf{2} \mid$ & 0.07 \\
\hline 4_5_2.5 & 343.0474 & $3|1| 2 \mid$ & 0.11 & 343.0474 & $\mathbf{1}|\mathbf{3}| \mathbf{2} \mid$ & 0.06 \\
\hline 4_5_3.0 & 286.1275 & $3->1|2|$ & 0.18 & 327.1712 & $2|\mathbf{3}->\mathbf{1}|$ & 0.22 \\
\hline 4_r_1.0 & 343.0474 & $3|1| 2 \mid$ & 0.07 & 343.0474 & $\mathbf{3}|\mathbf{2}| \mathbf{1} \mid$ & 0.07 \\
\hline 4_7_1.5 & 332.933 & $3|1->2|$ & 0.12 & 343.0474 & $2|1| 3 \mid$ & 0.12 \\
\hline 4_7_2.0 & 286.1275 & $2|1->3|$ & 0.23 & 331.5104 & $2|\mathbf{1}->3|$ & 0.16 \\
\hline 4_7_2.5 & 286.1275 & $2|3->1|$ & 0.06 & 316.4339 & $2|1->3|$ & 0.05 \\
\hline 4_7_3.0 & $286.12^{r 75}$ & $2|3->1|$ & 0.06 & 308.8765 & $1->3|2|$ & 0.20 \\
\hline 6_1_1.0 & 594.6428 & $1|4->2| 5|3|$ & 0.74 & 594.6428 & $3|4->2| 1|5|$ & 0.29 \\
\hline 6_1_1.5 & 594.3352 & $5|4| 3->2|1|$ & 0.32 & 594.6428 & $3|1| 5|4->2|$ & 0.42 \\
\hline 6_1_2.0 & 438.5142 & $2|3->1| 5->4 \mid$ & 0.30 & 494.5922 & $5|3->4->2| 1 \mid$ & 0.49 \\
\hline 6_1_2.5 & 387.9904 & $3->1|5->4->2|$ & 0.29 & 459.2829 & $5->4->2|3->1|$ & 0.51 \\
\hline 6_1_3.0 & 387.6828 & $5->4|1->3->2|$ & 0.22 & 441.4709 & $5->4->2|3->1|$ & 0.50 \\
\hline 6_3_1.0 & 645.1667 & $3|2| 4|5| 1 \mid$ & 0.33 & 645.1667 & $3|4| 5|1| 2 \mid$ & 0.39 \\
\hline 6_3_1.5 & 594.3352 & $4|3->2| 5|1|$ & 0.39 & 596.3519 & $5|4->2| 3|1|$ & 0.28 \\
\hline 6_3_2.0 & 445.4363 & $3->5|2| 4->1 \mid$ & 0.36 & 515.8627 & $1->2|3->4| 5 \mid$ & 0.46 \\
\hline 6_3_2.5 & 387.9904 & $5->4->2|3->1|$ & 0.17 & 462.7753 & $5->4->2|3->1|$ & 0.44 \\
\hline
\end{tabular}


Table 1 (conntinued)

\begin{tabular}{|c|c|c|c|c|c|c|}
\hline \multirow{2}{*}{$\begin{array}{l}\text { Instance } \\
\text { name }\end{array}$} & \multicolumn{3}{|c|}{ RCTVRP } & \multicolumn{3}{|c|}{ FRCTVRP } \\
\hline & Obj Value & Nodes in routes & Time[s] & Obj Value & Nodes in routes & Time[s] \\
\hline 6_3_3.0 & 387.6828 & $1->3->2|5->4|$ & 0.31 & 444.0907 & $3->1|5->4->2|$ & 0.45 \\
\hline 6_5_1.0 & 594.6428 & $1|5| 4->2|3|$ & 0.20 & 594.6428 & $1|3| 4->2|5|$ & 0.43 \\
\hline 6_5_1.5 & 594.3352 & $4|5| 3->2|1|$ & 0.28 & 594.6428 & $4->2|1| 3|5|$ & 0.47 \\
\hline 6_5_2.0 & 387.9904 & $5->4->2|3->1|$ & 0.18 & 486.8541 & $5->4->2|3->1|$ & 0.51 \\
\hline 6_5_2.5 & 387.9904 & $3->1|5->4->2|$ & 0.23 & 453.9269 & $3->1|5->4->2|$ & 0.51 \\
\hline 6_5_3.0 & 387.6828 & $4->5|1->3->2|$ & 0.18 & 437.4531 & $5->4->2|3->1|$ & 0.52 \\
\hline 6_7_1.0 & 534.9608 & $4->2|5->1| 3 \mid$ & 0.27 & 534.9608 & $5->1|4->2| 3 \mid$ & 0.50 \\
\hline 6_7_1.5 & 445.4363 & $5->3|2| 1->4 \mid$ & 0.20 & 505.5844 & $1->2|3| 5->4 \mid$ & 0.53 \\
\hline 6_7_2.0 & 362.8849 & $1->2|5->3->4|$ & 0.31 & 417.5379 & $5->3->4|1->2|$ & 0.47 \\
\hline $6 \_7 \_2.5$ & 360.6816 & $1|5->3->4->2|$ & 0.36 & 389.7075 & $1|5->3->4->2|$ & 0.43 \\
\hline 6_7_3.0 & 358.6308 & $5->2|1->3->4|$ & 0.29 & 382.4555 & $1|5->3->4->2|$ & 0.31 \\
\hline 8_1_1.0 & 541.5618 & $7|5| 6->1|4| 3->2 \mid$ & 4.18 & 584.5117 & $7|4| 3|2->5| 1->6 \mid$ & 10.15 \\
\hline 8_1_1.5 & 487.5307 & $5|7| 3->2|1->6->4|$ & 1.05 & 536.7901 & $5|7| 1->6->4|3->2|$ & 3.22 \\
\hline 8_1_2.0 & 431.0115 & $3->2->5|7| 1->6->4 \mid$ & 0.93 & 472.3581 & $\eta|1->6->4| 3->2->5 \mid$ & 1.93 \\
\hline 8_1_2.5 & 416.9024 & $2->5|4->6->1| 7->3 \mid$ & 1.35 & 458.5821 & $3->2->5|7| 1->6->4 \mid$ & 2.84 \\
\hline 8_1_3.0 & 379.6434 & $5->1->6->4|77->3->2|$ & 2.58 & 427.3815 & $1->6->4|5| 7->3->2 \mid$ & 2.21 \\
\hline 8_3_1.0 & 658.3218 & $5|3| 2|6->4| 1|7|$ & 2.72 & 658.3218 & $\eta|3| 6->4|5| 1|2|$ & 2.87 \\
\hline 8_3_1.5 & 487.5307 & $1->6->4|7| 5|3->2|$ & 1.04 & 540.3774 & $5|1->6->4| 7|3->2|$ & 8.28 \\
\hline 8_3_2.0 & 431.0115 & $7|1->6->4| 3->2->5 \mid$ & 0.79 & 474.4815 & $1->6->4|3->2->5| \eta \mid$ & 2.83 \\
\hline 8_3_2.5 & 416.9024 & $2->5|4->6->1| 7->3 \mid$ & 3.52 & 459.9981 & $1->6->4|7| 3->2->5 \mid$ & 2.93 \\
\hline 8_3_3.0 & 384.2311 & $5|4->6->1| 77->3->2 \mid$ & 0.75 & 428.2584 & $5|1->6->4| 7->3->2 \mid$ & 2.63 \\
\hline 8_5_1.0 & 574.233 & $4|7| 2->5|1->6| 3 \mid$ & 1.12 & 574.233 & $\eta|3| 4|1->6| 2$-> $5 \mid$ & 2.47 \\
\hline 8_5_1.5 & 487.5307 & $1->6->4|7| 5|3->2|$ & 0.89 & 531.5638 & $1->6->4|5| 7|3->2|$ & 9.03 \\
\hline 8_5_2.0 & 431.0115 & $4->6->1|7| 3->2->5 \mid$ & 1.21 & 469.6237 & $3->2->5|\eta| 1->6->4 \mid$ & 2.68 \\
\hline 8_5_2.5 & 416.9024 & $7->3|5->2| 1->6->4 \mid$ & 1.23 & 456.7588 & $7|3->2->5| 1->6->4 \mid$ & 2.78 \\
\hline 8_5_3.0 & 379.6434 & $5->1->6->4|7->3->2|$ & 1.04 & 426.5219 & $5|7->3->2| 1->6->4 \mid$ & 2.71 \\
\hline 8_7_1.0 & 485.0425 & $3->2->5|4| 7|1->6|$ & 1.07 & 485.0425 & $6->1|4| 7|3->2->5|$ & 1.90 \\
\hline 8_7_1.5 & 416.9024 & $2->5|1->6->4| 7->3 \mid$ & 0.82 & 442.2885 & $7|3->2->5| 1->6->4 \mid$ & 2.24 \\
\hline 8_7_2.0 & 384.2311 & $7->3->2|5| 4->6->1 \mid$ & 0.64 & 433.0913 & $7->3->2|5| 1->6->4 \mid$ & 2.50 \\
\hline 8_7_2.5 & 327.7119 & $1->6->4|7->3->2->5|$ & 0.36 & 368.4427 & $y->3->2->5|1->6->4|$ & 1.23 \\
\hline 8_7_3.0 & 327.7119 & $7->3->2->5|4->6->1|$ & 0.47 & 358.2634 & $1->6->4|7->3->2->5|$ & 1.87 \\
\hline
\end{tabular}




\section{Table 2}

A comparison of the risk indexes obtained on instances from the set $R$ for the RCTVRP and FRCTVRP

\begin{tabular}{|c|c|c|c|c|c|c|c|c|c|c|c|c|c|}
\hline 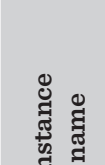 & 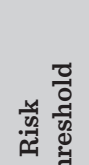 & 点 & & & & & & 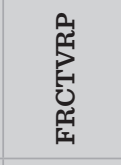 & & & & & \\
\hline & & $\begin{array}{c}\text { Travel } \\
\text { Distance }\end{array}$ & $\begin{array}{c}\text { Risk } \\
\text { indexes }\end{array}$ & & & & & $\begin{array}{c}\text { Travel } \\
\text { Distance }\end{array}$ & $\begin{array}{c}\text { Risk } \\
\text { indexes }\end{array}$ & & & & \\
\hline 4771.5 & 19995 & 332.93 & 13262.20 & 19561.70 & & & & 343.05 & 6785.72 & 4408.22 & 13262.20 & & \\
\hline $4 \_72.5$ & 33325 & 286.13 & 28548.90 & 6785.72 & & & & 286.13 & 23119.30 & 6785.72 & & & \\
\hline 4_7_3.0 & 39990 & 286.13 & 28548.90 & 6785.72 & & & & 286.13 & 23119.30 & 6785.72 & & & \\
\hline $6 \_11.5$ & 18720 & 594.34 & 8513.76 & 8483.65 & 15870.80 & 9148.45 & & 594.64 & 12464.40 & 9148.45 & 8513.76 & 11929.30 & \\
\hline $6 \_12.0$ & 24960 & 438.51 & 3355.24 & 24817.40 & 22184.10 & & & 463.23 & 9148.45 & 8513.76 & 24500.10 & & \\
\hline $6 \_13.0$ & 37440 & 387.68 & 35123.70 & 22184.10 & & & & 387.99 & 24817.40 & 25720.90 & & & \\
\hline 6_3_1.5 & 17856 & 594.34 & 8812.47 & 15318.80 & 8186.31 & 8938.95 & & 594.64 & 8186.31 & 12287.30 & 11889.10 & 8938.95 & \\
\hline 6_3_2.0 & 23808 & 445.44 & 23601.00 & 3380.85 & 23445.00 & & & 465.43 & 8186.31 & 20716.10 & 12691.60 & & \\
\hline 6_3_3.0 & 35712 & 387.68 & 34130.80 & 21986.00 & & & & 387.99 & 23884.80 & 25548.40 & & & \\
\hline 6_5_1.5 & 19584 & 594.34 & 8417.89 & 17268.10 & rar727.87 & 9707.14 & & 594.64 & 13039.70 & 12682.50 & ryrz'7.87 & 9707.14 & \\
\hline 6_5_3.0 & 39168 & 387.68 & 20853.70 & 37696.70 & & & & 387.99 & 25201.00 & 26099.30 & & & \\
\hline 6_7_1.5 & 22752 & 445.44 & 19944.80 & 5148.11 & 22241.70 & & & 445.44 & 21671.50 & 5148.11 & 22241.70 & & \\
\hline 6_7_3.0 & 45504 & 358.63 & 7523.10 & 43318.00 & & & & 360.68 & $9^{r} 707.14$ & 33090.50 & & & \\
\hline $8 \_11.0$ & 14738 & 541.56 & 11782.00 & 4321.40 & 14565.30 & 3837.09 & 14615.70 & 574.23 & 11782.00 & 3837.09 & 8694.49 & 11455.10 & 12498.10 \\
\hline $8 \_12.5$ & 29475 & 416.90 & 11455.10 & 24957.50 & 24333.20 & & & 431.01 & 21537.60 & 11782.00 & 17407.10 & & \\
\hline 8_13.0 & 35370 & 379.64 & 35145.80 & 30337.10 & & & & 384.23 & 17407.10 & 4321.40 & 30337.10 & & \\
\hline 8_3_2.5 & 29250 & 416.90 & 12136.80 & 24811.10 & 24081.10 & & & 431.01 & 17337.90 & 11692.10 & 22065.40 & & \\
\hline 8_3_3.0 & 35100 & 384.23 & 4454.37 & 24811.10 & 30532.20 & & & 384.23 & 4454.37 & 17337.90 & 30532.20 & & \\
\hline 8_5_2.5 & 29925 & 416.90 & 26164.90 & 14035.20 & $15^{r} 744.00$ & & & 431.01 & 11961.90 & 22541.60 & $157 r 74.00$ & & \\
\hline 8_5_3.0 & 35910 & 379.64 & 34331.50 & 31331.90 & & & & 384.23 & 4520.85 & 31331.90 & $15^{r} 774.00$ & & \\
\hline 8_7_1.0 & 17550 & 485.04 & 16907.50 & 5813.78 & 17538.10 & 13897.00 & & 485.04 & 17314.10 & 5813.78 & 17538.10 & 16907.50 & \\
\hline 8_7_1.5 & 26325 & 416.90 & 14290.70 & 20946.90 & 25535.60 & & & 431.01 & 17538.10 & 16907.50 & 20946.90 & & \\
\hline 8_7_2.0 & 35100 & 384.23 & 34437.90 & 3590.09 & 33059.90 & & & 384.23 & 34437.90 & 3590.09 & 20946.90 & & \\
\hline 8_7_3.0 & 52650 & 327.71 & 33059.9 & 42252.6 & & & & 327.71 & 20946.9 & 42252.6 & & & \\
\hline
\end{tabular}




\section{Table 3}

A comparison of the RCTVRP and FRCTVRP models on instances from the sets $\mathrm{V}, \mathrm{O}$ and $\mathrm{S}$

\begin{tabular}{|c|c|c|c|c|c|}
\hline \multirow{2}{*}{$\begin{array}{c}\text { Data } \\
\text { set }\end{array}$} & \multirow{2}{*}{$\begin{array}{c}\text { RISK } \\
\text { LEVEL }\end{array}$} & \multirow{2}{*}{ size } & \multicolumn{3}{|c|}{ RCTVRP } \\
\hline & & & Obj Value & Nodes in routes & Time[s] \\
\hline $\mathrm{V}$ & 1 & 22 & 874.70 & $20->14|1->6| 2->7|17->21| 19|4| 11->13|16| 15->18|9| 5|3->8->12| 10 \mid$ & 4128.71 \\
\hline $\mathrm{V}$ & 1.5 & 22 & 722.83 & $18|8->2->1->6| 12|19->16| 9->7|13| 11|17| 21->20->14|4->3| 10->5|15|$ & 449.17 \\
\hline $\mathrm{V}$ & 2 & 22 & 654.53 & $21->19|16| 15|4->3| 8|2->1->6| 17->20->18|12| 13->11|14| 10|9->7->5|$ & 3429.91 \\
\hline $\mathrm{V}$ & 2.5 & 22 & 655.24 & $15|5->7->16| 21->19|12| 13|8->14->17->20->18| 1->2->6|9| 4->3->11->10 \mid$ & 3086.47 \\
\hline $\mathrm{V}$ & 3 & 22 & 559.77 & $9->8|20->21->19| 12|14->16->17->18| 11->13|6->3->4| 15|1->2->5->7->10|$ & 3135.43 \\
\hline $\mathrm{O}$ & & 10 & 26.83 & $5->4->6|8->7->9| 2->1->3 \mid$ & 15.22 \\
\hline $\mathrm{O}$ & & 13 & 35.78 & $11->10->12|8->7->9| 5->4->6|2->1->3|$ & 10534.86 \\
\hline $\mathrm{O}$ & & 16 & 47.55 & $13->11->14->15|10| 12|2->3->1->4->5| 7->8->6->9 \mid$ & 3277.63 \\
\hline $\mathrm{O}$ & & 19 & 57.97 & $1->16|4->6->2| 10->13|12->8| 3->17->18->14|11->15| 7->9->5 \mid$ & 3062.91 \\
\hline $\mathrm{O}$ & & 21 & 70.71 & $15|1->4->3->2| 19->16->18->5|17| 12|7->6->8->9->10| 14->11->13->20 \mid$ & 2844.82 \\
\hline $\mathrm{S}$ & & 10 & 19.98 & $6->5->4|3->2->1| 9->8->7 \mid$ & 34.54 \\
\hline $\mathrm{S}$ & & 13 & 26.65 & $3->2->1|12->11->10| 6->5->4|9->8->7|$ & 12829.08 \\
\hline $\mathrm{S}$ & & 16 & 39.84 & $12->13->14->15|11| 9->10->8->7->6|5->4->3->2->1|$ & 2545.69 \\
\hline $\mathrm{S}$ & & 19 & 44.63 & $17->18->13|11->12->10| 7|16| 15->14|2->3| 8->9->4|6->5->1|$ & 2751.47 \\
\hline $\mathrm{S}$ & & 21 & 73.25 & $3->4->5|10->9->6->8->7| 18->19->20->11->12|16| 13|17| 2->1|14->15|$ & 2923.67 \\
\hline \multirow{2}{*}{$\begin{array}{l}\text { Data } \\
\text { set }\end{array}$} & \multirow{2}{*}{$\begin{array}{c}\text { RISK } \\
\text { LEVEL }\end{array}$} & \multirow{2}{*}{ size } & \multicolumn{3}{|c|}{ FRCTVRP } \\
\hline & & & Obj Value & Nodes in routes & Time[s] \\
\hline $\mathrm{V}$ & 1 & 22 & 940.62 & $11|3| 5|13| 19|21| 18->15|12| 20|9->7| 4|14| 8->2->6->10|1| 16|17|$ & 744.64 \\
\hline $\mathrm{V}$ & 1.5 & 22 & 761.37 & $20->16|14| 11|7->9->10| 3->4->8|13| 12|21->17| 19|18->15| 5|1->2->6|$ & 1960.54 \\
\hline $\mathrm{V}$ & 2 & 22 & 701.71 & $15|5->7->9| 11->13|12| 8->6->1->2->10|17| 21|19| 16|3->4| 18->20->14 \mid$ & 2042.16 \\
\hline $\mathrm{V}$ & 2.5 & 22 & 710.80 & $19->17|21->20->18| 13|14| 16|5->7| 4->3->8|12| 11|15->10->9| 1->2->6 \mid$ & 3574.96 \\
\hline $\mathrm{V}$ & 3 & 22 & 602.92 & $21->19->14|15| 16|11->3->4->8| 10->7->5->9|12| 2->1->6|13| 17->20->18 \mid$ & 7434.60 \\
\hline $\mathrm{O}$ & & 10 & 31.50 & $2->1->3|8->7->9| 5->4->6 \mid$ & 43.91 \\
\hline $\mathrm{O}$ & & 13 & 44.77 & $8->7->9|10->11| 5->4->6|12| 2->1->3 \mid$ & 4920.04 \\
\hline $\mathrm{O}$ & & 16 & 51.62 & $7->8->6->9->10|12->13->11->14->15| 5|1->4->3->2|$ & 4729.92 \\
\hline $\mathrm{O}$ & & 19 & 68.23 & $4|13| 3->17|10->11->15| 5->9|6->2| 1|16->18->14| 7->8->12 \mid$ & 8780.10 \\
\hline $\mathrm{O}$ & & 21 & 98.77 & $2|15| 17|10| 19|18->16| 20|13->11->14| 12|5| 6->9->8->7|3->1->4|$ & 7836.38 \\
\hline $\mathrm{S}$ & & 10 & 22.34 & $3->2->1|9->8->7| 6->5->4 \mid$ & 53.91 \\
\hline $\mathrm{S}$ & & 13 & 31.87 & $6->5->4|7| 9->8|3->2->1| 12->11->10 \mid$ & 5358.31 \\
\hline $\mathrm{S}$ & & 16 & 61.10 & $13->12|5->4->3| 11|6| 2|1| 10->9->8->7|15->14|$ & 5167.44 \\
\hline $\mathrm{S}$ & & 19 & 55.10 & $18->17|1| 13|3->2| 12->11|15->14| 6->5->4|9->8| 16|7| 10 \mid$ & 5890.35 \\
\hline $\mathrm{S}$ & & 21 & 78.31 & $7->6|15->14| 11|12| 5->4->3|8->10->9| 13|20->19->18->17->16| 2->1 \mid$ & 9723.64 \\
\hline
\end{tabular}


Thus, for some of the instances the routes of the optimal solutions are the same for the RCTVRP and FRCTVRP and those nodes in routes for the FRCTVRP are marked in bold. Firstly, let us note that results for the instances with the risk threshold index RL1 (instances whose name ends with "1.0") are the same. However, it is noticeable that the larger the risk threshold value is, the difference between two models is more significant. This is not surprising since larger values of the risk threshold lead to more variations in routes that satisfy the threshold conditions. These computational results show that the considered two versions of the problem lead to different optimal solutions for many tested instances. Sometimes the number of routes is different (e.g. "4_7_1.5"). Note that the number of routes in the optimal solution for the FRCTVRP can be larger or equal to the number of routes in the case of the classical RCTVRP. Commonly in the case of different solutions, the number of routes remains the same, but the nodes are not grouped in the same way or the order of nodes is not the same. For example, instance "4_7_2.5" has different results for the RCTVRP and FRCTVRP. Even though both solutions have the same value of the total travel distance (286.1275), the difference is in the risk indexes of the routes. For the RCTVRP the risk indexes are: 28548.9 and 6785.72 , while for the newly presented FRCTVRP model optimal solution has the following risk indexes of the routes: 23119.3 and 6785.72 , since the difference is the permutation of nodes within one of the routes.

A more detailed comparison for the instances that had different optimal routes in the two versions of the problem is given in Table 2 . The first column contains the instance name, while the risk threshold constant $\mathrm{T}$ is given in the second column. Then, firstly for the RCTVRP, and then for the FRCTVRP, the following values are provided: overall travel distance and global risk indexes of all the routes in the optimal solution.

The strength of the newly introduced model is that it makes it possible to differentiate less risky routes among all the possible routes. The aim stays to find short overall travel distances, but this model represents a compromise between travel distance and risk as explained in Section 4. Taking into account seven instances (shown in bold) with the same travel distance values for optimal solutions in both models, the summarized risk indexes are 378358.29 in the case of the RCTVRP, while they are 340943.69 in the case of the FRCTVRP. Thus, it can be seen on these test instances that the risk can be reduced by using the FRCTVRP instead of the RCTVRP, even for the same travel distances. Thus, the FRCTVRP provides less risky routes and is more suitable for the cash-intransit sector.

Moreover, the difference between the two models is more notable for larger instances. Therefore, it makes it even more important to use the FRCTVRP model when dealing with larger, realistic instances. Additional computational experiments were performed on instances with size 22 from a data set V (also described in [19]), as well as the smaller instances with sizes 10, 13, 16, 19 and 21 from two data sets $\mathrm{S}$ and $\mathrm{O}$, specially designed for the RCTVRP in [20]. These results are presented in Table 3 . The first column contains the name of data set, followed by the risk level $(r l)$ applicable for the instances in the set V. The third column shows the size of each instance, and the rest of the table is as described for Table 1 . It can be seen from Table 3 that only two instances with size 10 have the same routes for the both models.

Note that the execution time difference is very dependent on the test instances when these two models are compared. However, these differences are not significant and these two models are similarly challenging for CPLEX solver.

\section{Conclusion}

In this paper we developed a fuzzy version of the Risk-constrained Cash-in-Transit Vehicle Routing Problem (RCTVRP). The proposed fuzzy version of the RCTVRP, namely the FRCTVRP, represents an improvement of the classical RCTVRP in such a way that performs adequate comparison among feasible solutions by using a fuzzy number instead of fixed risk threshold limit. The FRCTVRP model takes into account the value of the risk index of each route and the solutions with lower values of risk indexes on their routes are considered as better.

Moreover, two mixed integer program formulations of the newly proposed FRCTVRP are developed in the paper. In order to compare the classical RCTVRP and the newly proposed FRCTVRP a small example 
is presented in details. The given example demonstrates the advantage of the newly presented fuzzy model. Computational analysis performed by using CPLEX solver showed that the difference between the two models is significant especially for larger test instances. Presented results on used data sets indicate that the proposed FRCTVRP is more suitable for the applications where transport of valuable goods is performed compared to the classical RCTVRP from the literature, since obtained optimal solutions have

\section{References}

1. Bent, R., Van Hentenryck, P. A Two-Stage Hybrid Local Search for the Vehicle Routing Problem with Time Windows. Transportation Science, 2004, 38(4), 515530. https://doi.org/10.1287//trsc.1030.0049

2. Blažinskas, A., Lenkevičius, A., Misevičius, A. Modified Local Search Heuristics for the Symmetric Traveling Salesman Problem. Information Technology and Control, 2013, 42(3), 217-230. https://doi.org/10.5755/j01.itc.42.3.1301

3. Brito, J., Martínez, F. J., Moreno, J. A., Verdegay, J. L. An ACO Hybrid Metaheuristic for Close-Open Vehicle Routing Problems with Time Windows and Fuzzy Constraints. Applied Soft Computing, 2015, 32, 154-163. https://doi.org/10.1016/j.asoc.2015.03.026

4. Cao, E., Lai, M. The Open Vehicle Routing Problem with Fuzzy Demands. Expert Systems with Applications, 2010, 37(3), 2405-2411. https://doi.org/10.1016/j. eswa.2009.07.021

5. Cordeau, J. F., Gendreau, M., Laporte, G. A Tabu Search Heuristic for Periodic and Multi-Depot Vehicle Routing Problems. Networks: An International Journal, 1997, 30(2), 105-119.

6. Desrochers, M., Desrosiers, J., Solomon, M. A New Optimization Algorithm for the Vehicle Routing Problem with Time Windows. Operations Research, 1992, 40(2), 342-354. https://doi.org/10.1287/opre.40.2.342

7. Drakulić, D., Takači, A., Marić, M. New Model of Maximal Covering Location Problem with Fuzzy Conditions. Computing and Informatics, 2016, 35(3), 635-652.

8. Ebrahimnejad, A., Verdegay, J. L. A Survey on Models and Methods for Solving Fuzzy Linear Programming Problems. Fuzzy Logic in Its 50th Year, Springer, Cham, 2016, 327-368

9. Eksioglu, B., Vural, A. V., Reisman, A. The Vehicle Routing Problem: A Taxonomic Review. Computers \& In- better quality in terms of safer routes. The newly proposed model incorporates more precise evaluation of each route's safety risk into the model, making more realistic distinction among routes that are safe enough to be considered in the cash-in-transit sector

\section{Acknowledgement}

Research partially supported by Serbian Ministry of Education and Science under the grants No. 174010 and III 044006.

dustrial Engineering, 2009, 57(4), 1472-1483. https:// doi.org/10.1016/j.cie.2009.05.009

10. Ghannadpour, S. F., Noori, S., Tavakkoli-Moghaddam, R., Ghoseiri, K. A Multi-Objective Dynamic Vehicle Routing Problem with Fuzzy Time Windows: Model, Solution and Application. Applied Soft Computing, 2014, 14, 504527. https://doi.org/10.1016/j.asoc.2013.08.015

11. Golden, B. L., Raghavan, S., Wasil, E. A. (Eds.). The Vehicle Routing Problem: Latest Advances and New Challenges (Vol. 43). Springer Science \& Business Media, 2008. https://doi.org/10.1007/978-0-387-777r78-8

12. Güran, A., Uysal, M., Ekinci, Y., Güran, C. B. An Additive FAHP Based Sentence Score Function for Text Summarization. Information Technology and Control, 2017, 46(1), 53-69. https://doi.org/10.5755/j01.itc.46.1.13051

13. Herrera, F., Verdegay, J. L. Three Models of Fuzzy Integer Linear Programming. European Journal of Operational Research, 1995, 83(3), 581-593. https://doi. org/10.1016/0377-2217(93)E0338-X

14. Klir, G., Yuan, B. Fuzzy Sets and Fuzzy Logic (Vol. 4). New Jersey: Prentice Hall, 1995.

15. Kuo, R. J., Zulvia, F. E., Suryadi, K. Hybrid Particle Swarm Optimization with Genetic Algorithm for Solving Capacitated Vehicle Routing Problem With Fuzzy Demand-A Case Study on Garbage Collection System. Applied Mathematics and Computation, 2012, 219(5), 2574-2588. https://doi.org/10.1016/j.amc.2012.08.092

16. Mladenović, N., Todosijević, R., Urošević, D. Two Level General Variable Neighborhood Search for Attractive Traveling Salesman Problem. Computers \& Operations Research, 2014, 52, 341-348. https://doi.org/10.1016/j. cor.2013.04.015

17. Ngueveu, S. U., Prins, C., Calvo, R. W. A Hybrid Tabu Search for the M-Peripatetic Vehicle Routing Problem. 
In Matheuristics, Springer, Boston, MA, 2009, 253-266. https://doi.org/10.1007/978-1-4419-1306-7_11

18. Skandari, M. N., Ghaznavi, M. An Efficient Algorithm for Solving Fuzzy Linear Programming Problems. Neural Processing Letters, 2018, 1-20.

19. Talarico, L., Sörensen, K., Springael, J. Metaheuristics for the Risk-Constrained Cash-in-Transit Vehicle Routing Problem. European Journal of Operational Research, 2015, 244(2), 457-470. https://doi.org/10.1016/j. ejor.2015.01.040

20. Talarico, L., Springael, J., Sörensen, K., Talarico, F. A Large Neighbourhood Metaheuristic for the Risk-Constrained Cash-in-Transit Vehicle Routing Problem. Computers \& Operations Research, 2017, 78, 547-556. https://doi.org/10.1016/j.cor.2016.04.003
21. Tang, J., Pan, Z., Fung, R. Y., Lau, H. Vehicle Routing Problem with Fuzzy Time Windows. Fuzzy Sets and Systems, 2009, 160(5), 683-695. https://doi. org/10.1016/j.fss.2008.09.016

22. Teodorović, D., Pavković, G. The Fuzzy Set Theory Approach to the Vehicle Routing Problem when Demand at Nodes is Uncertain. Fuzzy Sets and Systems, 1996, 82(3), 307-317. https://doi.org/10.1016/01650114(95)00276-6

23. Zedeh L. Fuzzy Sets. Information and Control, 1965, 8(3), 338-353. https://doi.org/10.1016/S00199958(65)90241-X

24. Zimmermann, H. J. Fuzzy Set Theory and Its Applications. Springer Science \& Business Media, 2001. https://doi.org/10.1007/978-94-010-0646-0 